\title{
Who communicates with whom?
}

Santos LD (1)

(1) Center for the Study of Venoms and Venomous Animals (CEVAP), São Paulo State University (UNESP - Univ Estadual Paulista), Botucatu, São Paulo State, Brazil.

There are numerous communication channels in nature, whether by physical contact or intracellular mediators. Several signaling pathways related to human diseases - including Alzheimer's disease, envenomation by animal toxins, treatment for anorexia, clinical features of depression, pain and prevention of premature birth - have been extensively studied in recent years (1). Much is known about the main clinical features of such diseases. However, research and development of new drugs are ongoing due to lack of specificity or long-term inefficacy of the currently available drugs.

Bioprospecting for new active biomolecules is an extremely interesting area, since it isolates stable molecules from nature, which have been evolutively tested by their producers during natural selection. The strategy of bioprospecting novel molecules has been the subject of numerous scientific studies. Several sensitive high resolution analytical techniques have been employed to find these potential pharmaceuticals. Nevertheless, elucidating the three-dimensional structure and knowing the function of active biomolecules are not enough to understand the action mechanism of an envenomation, considering that the envenomation occurs by a synergism between molecules and toxins present in the venom and by their direct action on cells of the victim organism. Within this context, knowing the signaling pathways of each molecule is necessary to better understand the mechanisms involved in an envenomation and to improve the existing antivenom therapy.

The new era of bioprospecting is focused on investigating the specific receptors of plasmatic membranes that are target of numerous toxins and finding the ionic channels through which such toxins pass. Who communicates with whom? This is the question that should be asked when one thinks about a clinical feature of envenomation. Because when the signaling pathways affected by animal toxins are disclosed, it is possible to act more specifically on the inhibition or stimulation of physiological processes in general.

A group of highly conserved $G$ proteins is the key determinant of specificity and temporal characteristics of numerous signaling pathways for signal transduction mechanisms in organisms (2). Such proteins are high molecular weight complex molecules found in plasmatic membranes of mammals in general. $G$ proteins are composed of three distinct polypeptides $(\alpha, \beta$ and $\gamma$ ) and form a signal transduction complex. When the extracellular specific receptors of $G$ proteins recognize exogenous molecules (antigens), a modification occurs in protein subunits, which enables the intracellular signal transmission. In this sense, the first analytical strategy, concerning macromolecules biochemistry and development of more specific drugs, is to discover which specific receptors interact with animal toxins. 
Since several researchers isolate molecules and utilize them in biological assays without elucidating their specific action sites and their signaling pathways, investment has been wasted by the scientific community. Besides, this scientific mistake justifies the chasm between prospective laboratory research in toxinology and the application of such molecules in clinical trials.

\section{COPYRIGHT}

(C) CEVAP 2012

\section{SUBMISSION STATUS}

Received: April 9, 2012.

Accepted: April 10, 2012.

Full paper published online: May 31, 2012.

\section{CONFLICTS OF INTEREST}

The author declares no conflicts of interest.

\section{CORRESPONDENCE TO}

Lucilene Delazari dos Santos, CEVAP-UNESP, Caixa Postal 577, Fazenda Experimental Lageado, Rua José Barbosa de Barros, 1780, 18610-307, Botucatu, SP, Brasil. Phone: +55 3814 5555. Email: lucilene@cevap.org.br

\section{REFERENCES}

1. Hemley CF, McCluskey A, Keller PA. Corticotropin releasing hormone - a GPCR drug target. Curr Drug Targets. 2007;8(1):105-15.

2. Lundstrom, K. Latest development in drug discovery on $\mathrm{G}$ protein-coupled receptors. Curr Protein Pept Sci. 2006;7(5):465-70. 